\title{
INITIATION AND PROPAGATION OF INTERFACIAL DELAMINATION IN INTEGRATED THIN-FILM STRUCTURES
}

\author{
Haixia Mei, Shravan Gowrishankar, Kenneth M. Liechti, Rui Huang \\ Department of Aerospace Engineering and Engineering Mechanics \\ University of Texas at Austin \\ 1 University Station, C0600 \\ Austin, Texas 78712 \\ Phone: (512) 471-7558 \\ Fax: (512) 471-5500 \\ Email: ruihuang@mail.utexas.edu
}

\begin{abstract}
Interfacial delamination has been a major reliability issue for both BEoL and packaging systems. The failure is often due to poor adhesion of interfaces. Thus characterization of interfacial properties is critical for material selection and process control. Conventional methods for interfacial adhesion and fracture toughness measurements are generally based on linear elastic fracture mechanics. More detailed local measurements are required to fully characterize the interfaces based on a nonlinear cohesive interface model. With the experimentally determined interfacial properties, cohesive interface modeling can be set up to predict the initiation and evolution of interfacial failure in chip-package systems. In this study, two model systems are considered by approaches of both linear elastic fracture mechanics (LEFM) and cohesive interface modeling (CIM). First, for a brittle thin film on a compliant substrate, the initiation and propagation of delamination from the root of a channel crack is simulated. The effects of the cohesive strength and fracture toughness of the interface on channel cracking of thin films on compliant substrates are analyzed. Second, a four-point bend test is considered, in comparison with experimental measurements of the local crack opening displacements.
\end{abstract}

KEY WORDS: fracture, delamination, interface

\section{NOMENCLATURE}

D damage parameter (dimensionless)

E elastic modulus $\left(\mathrm{N} / \mathrm{m}^{2}\right)$

G energy release rate $\left(\mathrm{J} / \mathrm{m}^{2}\right)$

$\mathrm{K} \quad$ interface stiffness $\left(\mathrm{N} / \mathrm{m}^{3}\right)$

$\mathrm{d} \quad$ delamination width $(\mathrm{m})$

$\mathrm{h}$ thickness (m)

\section{Greek symbols}

$\Gamma \quad$ fracture toughness $\left(\mathrm{J} / \mathrm{m}^{2}\right)$

$\alpha \quad$ first Dundurs parameter (dimensionless)

$\beta \quad$ second Dundurs parameter (dimensionless)

$\delta \quad$ opening displacement (m)

$\psi \quad$ phase angle of mode mix (radian)

$\begin{array}{ll}v & \text { Poisson's ratio (dimensionless) } \\ \sigma & \text { stress }\left(\mathrm{N} / \mathrm{m}^{2}\right) \\ \tau & \text { shear stress }\left(\mathrm{N} / \mathrm{m}^{2}\right) \\ & \\ \text { Subscripts } \\ \mathrm{f} & \text { film } \\ \text { s } & \text { substrate } \\ \text { ss } & \text { steady state }\end{array}$

\section{INTRODUCTION}

Continuous scaling of devices and performance requires innovations in materials, processes, and designs for both backend-of-line (BEoL) interconnects and packaging structures. Mechanical reliability has been a limiting factor for implementation of new materials and processes. In particular, interfacial delamination has been observed as a major reliability issue for both BEoL and packaging systems [1-2]. Two approaches are commonly used for interfacial failure analyses. One is based on stresses acting on the interfaces, typically obtained from finite element analyses (FEA). A comparison of the stress values with interfacial strengths for different material and geometric designs can be used to make some engineering judgments on the design and reliability. The other approach is based on the principle of linear elastic fracture mechanics (LEFM), where stress intensity factors or energy release rates are calculated as the driving force of delamination and the failure criterion is established by comparing the driving force with the interface toughness. Neither approach can model crack nucleation, which is critically important for the reliability analysis of chip-package systems. In this paper we develop a nonlinear cohesive interface model that is capable of modeling both nucleation and growth of interfacial delamination in a unified manner.

The cohesive interface model does not require any preassumption of the initial crack size or location. Under a specific thermo-mechanical loading, each interface behaves like a nonlinear material, undergoing damage initiation and evolution as described by a nonlinear traction-separation law. Therefore, crack nucleation at critical interfaces can be predicted [3]. As a nonlinear fracture mechanics approach, the cohesive interface model can be used to simulate progressive 
failure under cyclic loadings (e.g., fatigue), and other nonlinear material properties (e.g., nonlinear viscoelastic and elastic-plastic) can be incorporated consistently and efficiently.

Standard implementations of cohesive interface elements are available in several commercial FEA packages (e.g., ABAQUS and ANSYS). However, practical applications of this method require sufficient knowledge of the interfacial material parameters including both toughness and strength. Accurate characterization of specific material interfaces with detailed traction-separation laws has only been accomplished on a limited scale. This is due to the fact that local observables such as crack opening displacements must be measured, and the increasingly small length scales in the chip-package systems present grand challenges for such measurements. Previously, an iterative method to determine the tractionseparation laws for sapphire/epoxy interfaces by measuring the local crack opening displacement with crack-opening interferometry was developed [4]. A direct method combining global measurement of J-integral (energy release rate) and local measurements of crack-opening displacements by digital image correlation has also been developed for steel/polyurea interfaces with rate-dependent traction-separation laws [5].

In this paper, the cohesive interface modeling methodology is developed to simulate nucleation and propagation of interfacial delamination in two model systems. First, for a brittle thin film on a compliant substrate, fracture of the thin film occurs by channel cracking, which may be accompanied by interfacial delamination. As an example, a SiN thin film on Kapton is considered. Second, a four-point bend test structure with a $\mathrm{Si}$ bilayer on a carrier beam is considered to illustrate the necessity of local measurements for characterization of the interfacial properties.

\section{GENERAL DESCRIPTION OF COHESIVE ZONE MODEL}

In his study of nonlinear fracture mechanics, Dugdale [6] observed that the plastic zone ahead of a crack tip in a thin sheet of mild steel was primarily a narrow strip of height comparable to the sheet thickness (localized plastic deformation, or necking), while the length of the strip was much longer. The elastic-plastic fracture problem was then modeled by an elastic plane-stress problem with a strip of plastic zone ahead of each crack tip. Barenblatt [7] generalized the plastic strip model to a cohesive zone model (CZM) in which the stress in the cohesive zone ahead of the crack is a function of the separation rather than a constant yield stress. Cottrell [8] put forward the concept of crack bridging as a unifying theory for fracture at various length scales, from atomic bond breaking in monolithic ceramics to fiber pull-out in composite materials. In each case, the microscopic mechanism of fracture and associated inelastic processes are represented by a bridging law that relates the face tractions in the bridging zone (or cohesive zone) to the relative separation displacements, as illustrated in Fig. 1. The essential features of crack bridging were reviewed by Bao and Suo [9], emphasizing their implications for strength and fracture resistance of ceramic matrix composites. The concept has also been widely used for modeling interfaces between elastic- plastic materials [10].
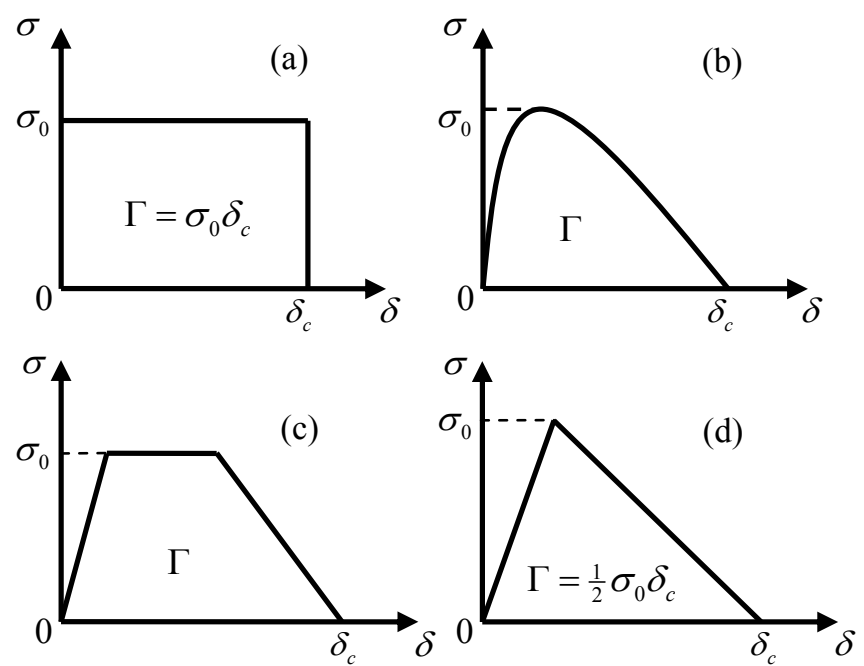

Fig. 1: Examples for the traction-separation relationship as the bridging law for the cohesive zone modeling: (a) constant traction for perfect plasticity [6]; (b) smooth nonlinear [11]; (c) trapezoidal [12, 13]; (d) bilinear or triangular [14].

Unlike LEFM where the microscopic mechanisms of fracture are essentially ignored (all material aspects are lumped into one parameter, fracture toughness), the bridging law or the traction-separation relation in the cohesive zone model depends on the material and the associated fracture mechanism. For example, in an ideally brittle material, fracture occurs by atomic bond breaking, for which a bridging law may be derived from an interatomic bond potential. For metals, however, the fracture mechanism is different, with large plastic deformation (local necking) and void nucleation, growth, and coalescence ahead of the crack tip. The bridging law, $\sigma(\delta)$, may be derived from detailed micromechanics models or may be determined experimentally $[11,12]$. On the other hand, relatively simple bridging laws are often used in theoretical and numerical analyses. For example, the Dugdale model assumes a constant traction in the bridging zone [6]. A triangular or trapezoidal shaped traction-separation curve is frequently used in practice [12-14]. In any case, the maximum stress $\sigma_{0}$ and the critical displacement $\delta_{c}$ are the two most important parameters that characterize the traction-separation relation. Given a traction-separation law, the fracture toughness (i.e., energy dissipation per unit area of the crack) can be obtained as

$$
\Gamma=\int_{0}^{\delta_{c}} \sigma(\delta) d \delta \sim \sigma_{0} \delta_{c} .
$$

For brittle fracture, $\sigma_{0}$ is roughly one order of magnitude lower than the elastic modulus $\left(\sigma_{0} \sim 10 \mathrm{~N} / \mathrm{m}^{2}\right)$ and $\delta_{\mathrm{c}}$ is comparable to the interatomic distance $\left(\sim 10^{-10} \mathrm{~m}\right)$. Thus, an order-ofmagnitude estimate gives that $\Gamma \sim 1 \mathrm{~J} / \mathrm{m}$, which is essentially the surface energy per unit area of the solid. For metals, $\sigma_{0}$ corresponds to the yield stress $\left(\sim 10^{8} \mathrm{~N} / \mathrm{m}^{2}\right)$ and $\delta_{c}$ is typically in the order of $10^{-6} \mathrm{~m}$ (depending on the microstructures), which lead to a fracture energy, $\Gamma \sim 10^{2} \mathrm{~J} / \mathrm{m}^{2}$, much higher 
than the surface energy.

Cohesive zone modeling is particularly suitable for adhesion and debonding of interfaces between two dissimilar materials [10], where the constituent materials can be either linear elastic or elastic-plastic. Depending on the material systems, the maximum stress of the interfacial bridging law $\sigma_{0}$ can be either small or large compared to the yield stresses of the constituent materials. When $\sigma_{0}$ is greater than the yield stress, plastic deformation in the constituent material occurs during interfacial fracture, and the total energy of fracture is greater than the intrinsic fracture energy of the interface $(\Gamma \sim$ $\sigma_{0} \delta_{c}$ ). Therefore, the effect of plasticity can be analyzed by coupling the bridging law for the interface with continuum elastic-plastic models for the constituent materials [15].
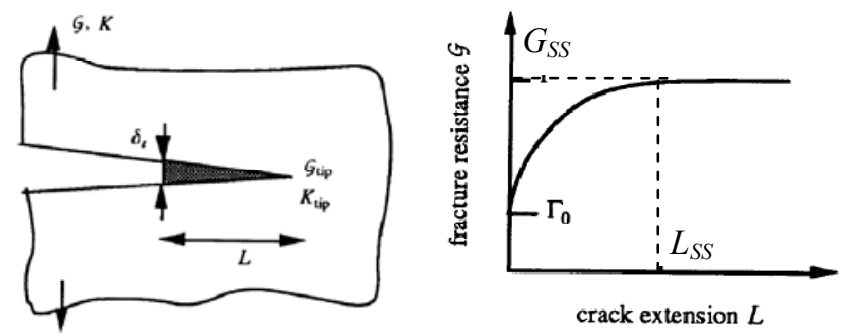

Fig. 2: A schematic of cohesive zone and R-curve (small-scale bridging model [9]).

Small-scale bridging. The size of the cohesive zone ahead of the crack tip may be estimated under the condition of small scale bridging (similar to the small-scaling yielding condition in LEFM). Assume that the cohesive zone size is small compared to the crack size, $L<<a$. In this case, the external load can be represented by the stress intensity factor $K$ or energy release rate $G$, ignoring the details in the cohesive zone. The crack starts to grow when $G=\Gamma_{0}$, where $\Gamma_{0}$ is the fracture energy for crack initiation. As the crack front advances, a cohesive zone develops (see Fig. 2). An application of the Jintegral along a contour at the boundary of the cohesive zone gives that

$$
G=J=G_{t i p}+\int_{0}^{\delta_{t}} \sigma(\delta) d \delta
$$

where $\delta_{t}$ is the opening displacement at the end of the cohesive zone and $G_{t i p}$ is the energy release rate at the crack tip. While fracture at the tip of the bridging zone occurs with $G_{\text {tip }}=\Gamma_{0}$, the energy dissipation in the cohesive zone requires a larger energy release rate $G>\Gamma_{0}$, as the size of the cohesive zone increases. The length $L$ increases as the applied load increases, until a steady state is reached when the separation at the end of the cohesive zone reaches the critical value, $\delta_{t}=\delta_{c}$. Subsequently, the cohesive zone size remains a constant, $L=$ $L_{\mathrm{SS}}$, as the crack grows. The required energy release rate for the steady state crack growth is thus:

$$
G_{S S}=\Gamma_{0}+\int_{0}^{\delta_{c}} \sigma(\delta) d \delta
$$

The so-called resistance curve (R-curve) is obtained by plotting the energy release rate as a function of the crack extension, in which $G$ increases from $\Gamma_{0}$ to $G_{S S}$ (Fig. 2). To determine the R-curve and the steady state cohesive zone size, a boundary value problem has to be solved. Within the cohesive zone, the face traction is related to the opening displacement by the traction-separation law. The outer boundary condition is given by the $K$ field corresponding to the applied energy release rate $G$, under the condition of small-scale bridging. A dimensional analysis [9] leads to a scaling law for the steady-state cohesive zone size:

$$
L_{S S} \sim \frac{E G_{S S}}{\sigma_{0}^{2}} .
$$

The small scale bridging condition is satisfied when the crack size $a>L_{\mathrm{SS}}$.

Large-scale bridging. The small scale bridging condition is rarely satisfied in practice for composites and interfaces. When large scale bridging occurs, the R-curve depends sensitively on the specimen geometry and thus cannot be used to predict the strength and load carrying capacity of components of different sizes and geometry. A full stress analysis coupling the specimen geometry and the bridging law must be carried out to predict the mechanical properties including the resistance to fracture [9]. In the case of interfacial fracture, the traction-separation relation must be experimentally characterized and then incorporated in the stress analysis as part of the material properties to analyze the interfacial reliability.

\section{IMPLEMENTATION OF COHESIVE ZONE MODEL}

The cohesive zone model has been implemented in the commercial finite element package, ABAQUS [16]. In particular, cohesive elements are defined along the potential paths of crack growth (e.g., a bimaterial interface). The constitutive properties of the cohesive elements are specified in terms of the traction-separation law. Several types of traction-separation laws have been implemented, and selfdeveloped user subroutines may be incorporated for the cohesive elements with non-standard traction-separation laws. In this study, we adopt the bilinear traction-separation law (Fig. 3) for interfacial fracture.

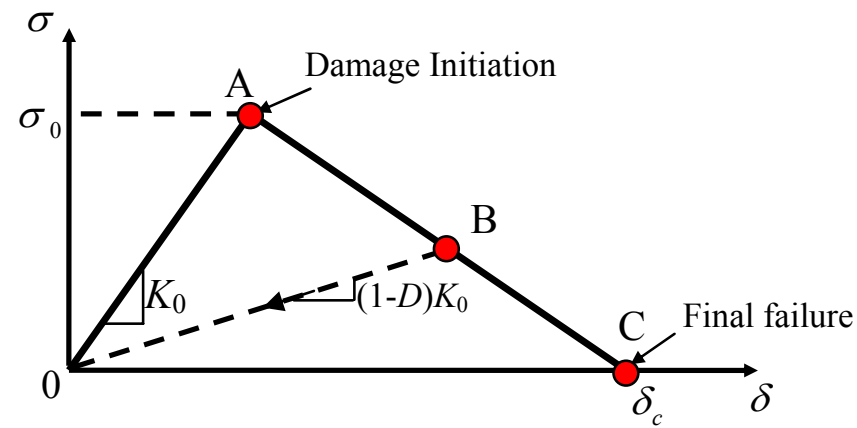

Fig. 3. Illustration of the bilinear traction-separation law in ABAQUS.

First, consider opening mode (mode I) fracture of an interface. Figure 3 shows the parameters required to define the 
interfacial elements: an initial elastic stiffness $K_{0}$, a cohesive strength $\sigma_{0}$, and a critical separation $\delta_{c}$. Subject to an opening stress $\sigma$, the interface first opens elastically with the initial stiffness until the stress reaches the cohesive strength of the interface $\left(\sigma=\sigma_{0}\right)$, at which point damage initiation occurs. A damage parameter $D$ is used to describe the state of the interface, which evolves from 0 to 1 based on a damage evolution rule:

$$
D=\frac{\delta_{c}\left(\delta_{\max }-\delta_{0}\right)}{\delta_{\max }\left(\delta_{c}-\delta_{0}\right)},
$$

where $\delta_{0}=\sigma_{0} / K_{0}$ is the critical separation for damage initiation and $\delta_{\max }$ is the maximum separation for the interface element over the entire loading history. When the interface element is partly damaged $(0<D<1)$, the opening stress is related to the opening displacement linearly as

$$
\sigma=(1-D) K_{0} \delta
$$

Eq. (6) applies for both loading and unloading. During loading, $\delta_{\max }=\delta$, and thus the damage parameter $D$ increases as the opening displacement increases. Combining Eq. (5) and (6) gives that the stress decreases linearly with the displacement $\delta\left(\delta_{0} \leq \delta \leq \delta_{c}\right)$ :

$$
\sigma=\sigma_{0} \frac{\delta_{c}-\delta}{\delta_{c}-\delta_{0}}
$$

When $\delta \geq \delta_{c}, D=1$ and $\sigma=0$, indicating that the interface element is fully fractured. During unloading (e.g., from B in Fig. 3), $\delta_{\max }$ remains as a constant and so does $D$. Therefore, the stress decreases linearly as the opening displacement decreases, with the slope $K=(1-D) K_{0}$ as illustrated by the dashed line in Fig. 3.

For interfacial delamination between two dissimilar materials, mode mix is commonly observed. In ABAQUS, the traction-separation laws for the opening and shearing fracture modes (modes I, II, and III) can be defined separately, each with a set of similar parameters as the opening mode. Under a mixed mode condition, however, the criteria for damage initiation and final failure must be specified taking into account the effect of mode mix. Several different criteria have been implemented in ABAQUS. For the present study, we adopt an elliptic form for the damage initiation criterion, namely

$$
\left(\frac{\langle\sigma\rangle}{\sigma_{0}}\right)^{2}+\left(\frac{\tau}{\tau_{0}}\right)^{2}=1,
$$

where $\tau_{0}$ is the shear strength of the interface, $\langle\sigma\rangle=\sigma$ if $\sigma>0$ (tension) and $\langle\sigma\rangle=0$ otherwise. By using the Macauley bracket we assume that compression does not cause damage. As a result of the mixed-mode damage initiation criterion, the critical magnitude of the traction vector depends on the ratio between the shear and normal tractions. Define a phase angle for the local mode mix as

$$
\tan \psi=\frac{\tau}{\langle\sigma\rangle} .
$$

The magnitude of the effective traction vector is

$$
\sigma_{m}=\sqrt{\langle\sigma\rangle^{2}+\tau^{2}}=\frac{\langle\sigma\rangle}{\cos \psi}=\frac{\tau}{\sin \psi} .
$$

By Eqs. (8)-(10), the critical traction magnitude for damage initiation is

$$
\sigma_{m 0}=\sigma_{0}\left(\cos ^{2} \psi+\frac{\sigma_{0}^{2}}{\tau_{0}^{2}} \sin ^{2} \psi\right)^{-1 / 2}
$$

To describe evolution of the damage parameter $D$ under a combination of normal and shear deformation across the interface, an effective displacement is defined as:

$$
\delta_{m}=\sqrt{\left\langle\delta_{n}\right\rangle^{2}+\delta_{s}^{2}}
$$

where $\delta_{n}$ and $\delta_{s}$ are normal and shear displacement, respectively. The damage parameter is then determined by an evolution rule similar to Eq. (5):

$$
D=\frac{\delta_{m c}\left(\delta_{\max }-\delta_{m 0}\right)}{\delta_{\max }\left(\delta_{m c}-\delta_{m 0}\right)}
$$

where $\delta_{m c}$ is the critical effective displacement, depending on the mode mix and the final failure criterion. In the present study, we adopt the energy-based failure criterion:

$$
\frac{G_{I}}{\Gamma_{I}}+\frac{G_{I I}}{\Gamma_{I I}}=1
$$

where $\Gamma_{I}$ and $\Gamma_{I I}$ are the fracture toughness under pure mode I (opening) and mode II (shearing) conditions, respectively, $G_{I}$ and $G_{I I}$ are the work done by the tractions in normal and shear directions, namely

$$
\begin{aligned}
& G_{I}=\int_{0}^{\delta_{n}} \sigma d \delta_{n}, \\
& G_{I I}=\int_{0}^{\delta_{s}} \tau d \delta_{s} .
\end{aligned}
$$

Similar to Eq. (6) for the opening mode, the shear traction is related to the shear displacement as

$$
\tau=(1-D) K_{0} \delta_{s}
$$

where the initial stiffness $\left(K_{0}\right)$ is assumed to be identical for the normal and shear deformation [14]. Thus, the ratio between the two tractions is identical to the corresponding ratio between the two displacements, and by Eq. (12) we have

$$
\delta_{m}=\frac{\left\langle\delta_{n}\right\rangle}{\cos \psi}=\frac{\delta_{s}}{\sin \psi} .
$$

Substitution of Eq. (18) into Eq. (13) leads to 


$$
D=\frac{\delta_{m c}\left(\left\langle\delta_{n}\right\rangle-\delta_{m 0} \cos \psi\right)}{\left(\delta_{m c}-\delta_{m 0}\right)\left\langle\delta_{n}\right\rangle}=\frac{\delta_{m c}\left(\delta_{s}-\delta_{m 0} \sin \psi\right)}{\left(\delta_{m c}-\delta_{m 0}\right) \delta_{s}} .
$$

Inserting Eq. (19) into Eq. (6) and Eq. (17), we obtain the tractions

$$
\begin{aligned}
& \sigma=\frac{K_{0} \delta_{m 0}}{\delta_{m c}-\delta_{m 0}}\left(\delta_{m c} \cos \psi-\left\langle\delta_{n}\right\rangle\right), \\
& \tau=\frac{K_{0} \delta_{m 0}}{\delta_{m c}-\delta_{m 0}}\left(\delta_{m c} \sin \psi-\delta_{s}\right),
\end{aligned}
$$

both of which decrease linearly with the corresponding displacement components. At the point of final failure, $\left\langle\delta_{n}\right\rangle=\delta_{m c} \cos \psi$ and $\delta_{s}=\delta_{m c} \sin \psi$. Moreover, the work done by the tractions are simply

$$
\begin{aligned}
& G_{I}=\frac{1}{2} \sigma_{m 0} \delta_{m c} \cos ^{2} \beta, \\
& G_{I I}=\frac{1}{2} \sigma_{m 0} \delta_{m c} \sin ^{2} \beta .
\end{aligned}
$$

By the mixed-mode failure criterion in (14), we obtain that

$$
\begin{aligned}
& \delta_{m c}=\left(\frac{\sigma_{m 0}}{2 \Gamma_{I}} \cos ^{2} \psi+\frac{\sigma_{m 0}}{2 \Gamma_{I I}} \sin ^{2} \psi\right)^{-1} \\
& =\delta_{n c}\left(\cos ^{2} \psi+\frac{\Gamma_{I}}{\Gamma_{I I}} \sin ^{2} \psi\right)^{-1}\left(\cos ^{2} \psi+\frac{\sigma_{0}^{2}}{\tau_{0}^{2}} \sin ^{2} \psi\right)^{1 / 2}
\end{aligned}
$$

The total mixed-mode fracture energy (per unit area) is then

$$
\Gamma=G_{I}+G_{I I}=\frac{1}{2} \sigma_{m 0} \delta_{m c}=\Gamma_{I}\left(\cos ^{2} \psi+\frac{\Gamma_{I}}{\Gamma_{I I}} \sin ^{2} \psi\right)^{-1} .
$$

Therefore, under a mixed-mode loading, both the strength (Eq. 11) and toughness (Eq. 25) depend on the phase angle of mode mix. In addition, the mixed-mode interface strength depends on the normal and shear strength, and the mixedmode interface toughness depends on the mode-I and mode-II toughness. Together, at least five parameters are needed to describe the interfacial fracture: one stiffness $\left(K_{0}\right)$, two strengths $\left(\sigma_{0}\right.$ and $\left.\tau_{0}\right)$, and two toughnesses $\left(\Gamma_{I}\right.$ and $\left.\Gamma_{I I}\right)$.

It shall be noted that the phase angle for mode mix as defined in Eq. (9) can be different from the phase angle defined in LEFM. In particular, for an interfacial crack in LEFM, due to the oscillatory singularity at the crack tip, an arbitrary length scale must be specified to define the phase angle [17, 18], except for the cases when the index of oscillatory singularity is zero (e.g., when the two materials across the interface have identical elastic properties). In the cohesive zone model, the phase angle is defined locally at each point or for each interface element in the finite element analysis. Therefore, the mode mix may vary along the interface (from element to element) and may change during the loading process. For clarity, we call the mode mix of each interface element the local mode mix and the mode mix by
LEFM the global mode mix. Furthermore, the energy release rates in Eq. (15-16) and the failure criterion in Eq. (14) are all defined locally for each interface element.
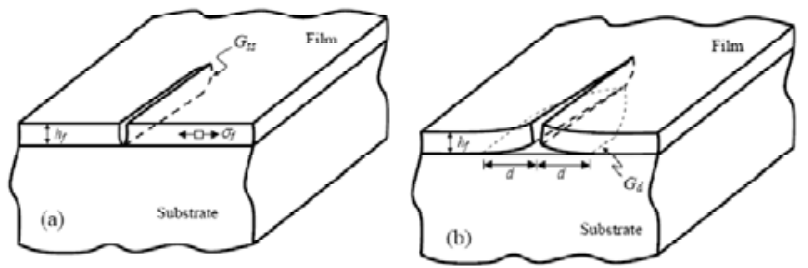

Fig. 4: (a) A channel crack with no interfacial delamination; (b) A channel crack with stable interfacial delamination of width $d$ on both sides.

\section{CHANNEL CRACKING WITH DELAMINATION}

As illustrated in Fig. 4a, assuming no interfacial delamination, the energy release rate for steady-state growth of a channel crack in an elastic film bonded to a thick elastic substrate is

$$
G_{s s}=Z(\alpha, \beta) \frac{\sigma_{f}^{2} h_{f}}{\bar{E}_{f}},
$$

where $\sigma_{f}$ is the tensile stress in the film, $h_{f}$ is the film thickness, and $\bar{E}_{f}=E_{f} /\left(1-v_{f}^{2}\right)$ is the plane-strain modulus of the film with Young's modulus $E_{f}$ and Poisson's ratio $v_{f}$. The dimensionless coefficient $Z$ depends on the elastic mismatch between the film and the substrate, through the Dundurs parameters

$$
\begin{gathered}
\alpha=\frac{\bar{E}_{f}-\bar{E}_{s}}{\bar{E}_{f}+\bar{E}_{s}}, \\
\beta=\frac{\bar{E}_{f}\left(1-v_{f}\right)\left(1-2 v_{s}\right)-\bar{E}_{s}\left(1-v_{s}\right)\left(1-2 v_{f}\right)}{2\left(1-v_{f}\right)\left(1-v_{s}\right)\left(\bar{E}_{f}+\bar{E}_{s}\right)} .
\end{gathered}
$$

When the film and the substrate have identical elastic moduli, we have $\alpha=\beta=0$ and $Z=1.976$. The value of $Z$ increases rapidly for a stiff film on a relatively compliant substrate $\left(\bar{E}_{f}>\bar{E}_{s}\right.$ and $\left.\alpha>0\right)$. For a $\operatorname{SiN}$ film $\left(E_{f}=310\right.$ $\left.\mathrm{GPa}, v_{f}=0.27\right)$ on Kapton $\left(E_{s}=5 \mathrm{GPa}, v_{s}=0.32\right)$, we have $\alpha=0.9672$ and $Z=17.64$. Consequently, the driving force for channel cracking can be significantly higher when the film is bonded to a more compliant substrate.

Now consider an interfacial crack emanating from the root of a channel crack, as shown in Fig. 4b. For a long, straight channel crack, we assume a steady state far behind the channel front, where the interfacial crack has a finite width $d$. The energy release rate for the interfacial crack takes the form

$$
G_{d}=Z_{d}\left(\frac{d}{h_{f}}, \alpha, \beta\right) \frac{\sigma_{f}^{2} h_{f}}{\bar{E}_{f}},
$$


where $Z_{d}$ is a dimensionless function that can be determined from a two-dimensional finite element model [19,20]. Depending on the elastic mismatch and the interface toughness, there can be no interfacial delamination, stable delamination or unstable delamination, which has been summarized in a diagram [20]. For a stiff film on a compliant substrate, it was found that delamination always occurs by the LEFM criterion. Stable delamination with a finite width $d$ is predicted when the interface toughness is greater than a critical value, $\Gamma_{i}>\sigma_{f}^{2} h_{f} /\left(2 \bar{E}_{f}\right)$. With the stable delamination, the effective driving force for the growth of channel cracks is further enhanced. To account for the influence of interfacial delamination on both the fracture driving force and the fracture resistance, an effective energy release rate for channel cracking is defined as [20]

$$
G_{s s}^{e f f}=Z_{e f f}\left(\bar{\Gamma}_{i}, \alpha, \beta\right) \frac{\sigma_{f}^{2} h_{f}}{\bar{E}_{f}},
$$

where the dimensionless coefficient $Z_{\text {eff }}$ depends on the normalized interface toughness, $\bar{\Gamma}_{i}=\bar{E}_{f} \Gamma_{i} /\left(\sigma_{f}^{2} h_{f}\right)$, in addition to the Dundurs parameters for the elastic mismatch. Using the effective energy release rate, the condition for the steady-state channel cracking is simply a comparison between $G_{s s}^{\text {eff }}$ and the film toughness $\Gamma_{f}$, the latter being a constant independent of the interface.
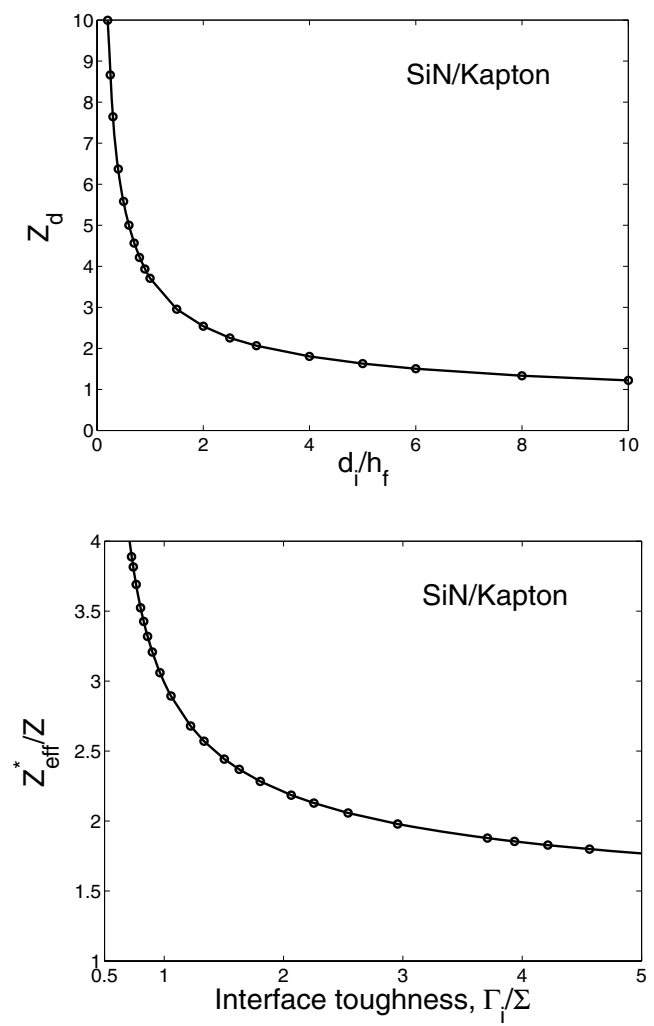

Fig. 5: (a) Normalized energy release rate of interfacial delamination as a function of the normalized delamination width. (b) Effective energy release rate for channel cracking as a function of the normalized interface toughness.
Specifically, for a SiN film deposited on Kapton, Fig. 5a plots the normalized energy release rate $Z_{d}$ as a function of the delamination width, and Fig. $5 \mathrm{~b}$ plots the normalized effective energy release rate, $Z_{e f f} / Z(\alpha, \beta)$, as a function of the normalized interface toughness. The effective driving force increases as the normalized interface toughness deceases. At the limit of high interface toughness $\left(\bar{\Gamma}_{i} \rightarrow \infty\right)$, the delamination width $d \rightarrow 0$ and $Z_{e f f} / Z \rightarrow 1$, recovering the case of channel cracking with no delamination.

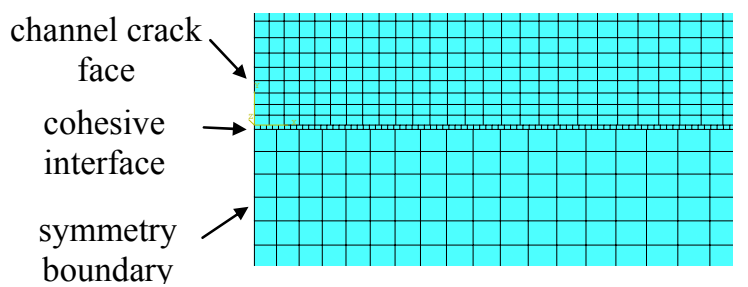

Fig. 6: An example finite element mesh, with cohesive elements for the interface between the film and substrate.

Next we simulate the initiation and propagation of the interface crack using the cohesive interface model in ABAQUS. As shown in Fig. 6, a layer of cohesive elements is used to model the interface between the film and the substrate, using the triangular traction-separation law (Fig. 3). For simplicity, we ignore the effect of mode mix in the present study by taking $\sigma_{0}=\tau_{0}$ and $\Gamma_{I}=\Gamma_{I I}=\Gamma$.

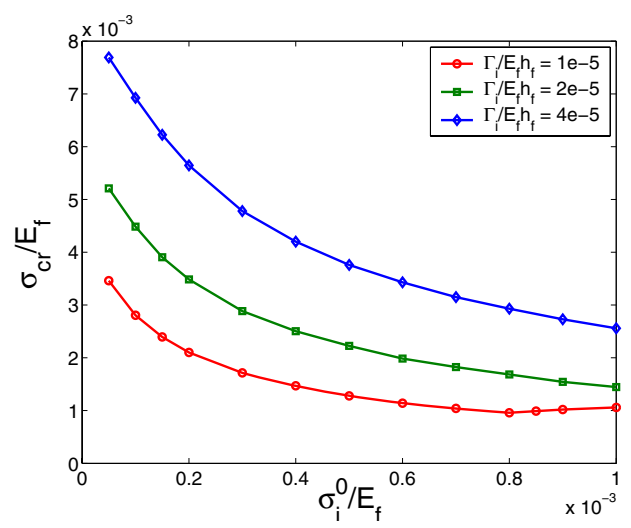

Fig. 7: Critical stress for interfacial crack initiation as a function of the interface strength.

Figure 7 shows the critical stress for initiation of an interfacial crack from the root of a channel crack as a function of the normalized interface strength, $\sigma_{0} / \bar{E}_{f}$, for different values of the interface toughness. By LEFM, as the energy release rate for interface delamination approaches infinity as the interface crack length $d \rightarrow 0$ (Fig. 5a), the critical stress for the crack initiation is essentially zero. However, by the cohesive interface model, the critical stress for delamination crack initiation is a finite value that depends on both the 
interface toughness and strength. As expected, the critical stress increases as the interface toughness increases. The effect of interface strength however is two-fold. First, the criterion for damage initiation of the cohesive elements requires that the stress at the interface reaches the strength. Thus, the critical stress for the damage initiation increases with the interface strength, similar to the strength criterion. On the other hand, to initiate an interfacial crack, the first cohesive element must be fully damaged and meanwhile a cohesive zone must be developed ahead of the crack tip. The total energy required for the crack initiation thus includes that for the crack (equals the fracture toughness $\Gamma$ ) and that dissipated in the cohesive zone. Therefore, the critical stress for the crack initiation also depends on the size of the cohesive zone, which scales with $\bar{E}_{f} \Gamma / \sigma_{0}^{2}$ [13]. For the same toughness, the cohesive zone size decreases as the interface strength increases. As shown in Fig. 7 , when the interface strength is relatively low, the cohesive zone size is large (large-scale bridging) and the critical stress for crack initiation decreases as the strength increases for the same toughness. The trend seems to be reversed when the interface strength is high, where the cohesive zone size approaches the limit of small-scale bridging. Under the condition of small-scale bridging, the results should approach the LEFM predictions (toughness criterion), which is independent of the interface strength. The slight increase of the critical stress may be attributed to the increase of the damage initiation stress.

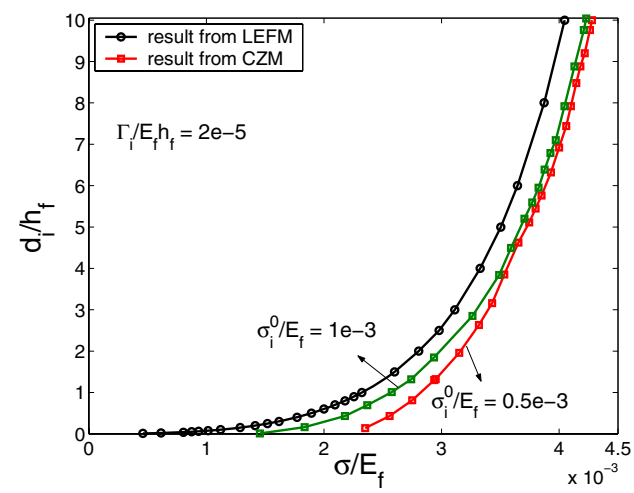

Fig. 8: Arrested delamination width as a function of the film stress.

After initiation, the interfacial crack grows stably as the film stress increases. Figure 8 compares the delamination length from the LEFM and the cohesive interface models. By LEFM, the critical stress is zero and the crack length increases immediately as the film stress increases. By the cohesive interface model, the interfacial crack length remains zero until the film stress reaches the critical value, beyond which the crack length increases with the film stress. For the same interface toughness, the crack length increases as the interface strength increases, which again can be attributed to the effect of the interface strength on the cohesive zone size. With larger cohesive strength and consequently smaller cohesive zone, the results from the cohesive interface model compare more closely to the LEFM results. Thus, the growth of the interfacial crack depends on both the toughness and strength of the interface.

\section{FOUR-POINT CARRIER BEND TEST}

A schematic of the four-point bend test with a steel carrier beam is shown in Fig. 9. A bilayer Si specimen was bonded to the carrier beam using a double-sided carbon tape. The thickness of the carrier beam is $h_{1}$, and that of each Si layer is $h_{\mathrm{s}}$. An initial crack of length $a_{0}$ was introduced along the interface between the two Si layers.

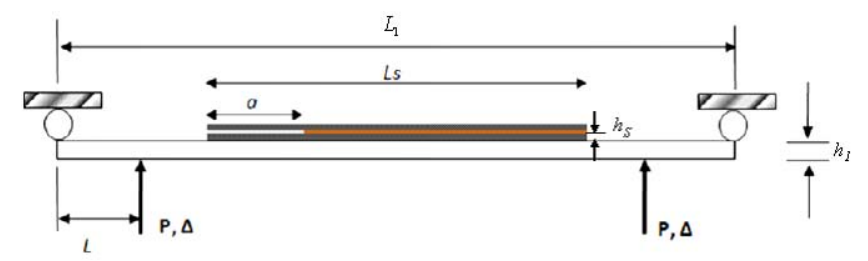

Fig. 9: Schematic of four-point carrier bend test.

A finite element model was developed to simulate the carrier bend testing, with a layer of cohesive interface elements tied in between of the two $\mathrm{Si}$ beams. The geometric parameters of the model are: $h_{\mathrm{s}}=0.32 \mathrm{~mm}, h_{1}=4 \mathrm{~mm}, L_{\mathrm{s}}=40$ $\mathrm{mm}, L_{1}=84 \mathrm{~mm}$, and $L=11.5 \mathrm{~mm}$. The width of the beams is $b=5 \mathrm{~mm}$. Linear elastic material properties are assumed for both the $\mathrm{Si}$ and steel, with $E_{\mathrm{Si}}=168 \mathrm{GPa}, E_{\text {steel }}=210 \mathrm{GPa}, v_{\mathrm{Si}}$ $=0.22$, and $v_{\text {steel }}=0.27$. The parameters specified for the interface elements are: $K_{0}=10^{7} \mathrm{MPa} / \mathrm{mm}, \sigma_{0}=\tau_{0}=20$ $\mathrm{MPa}, \Gamma_{I}=\Gamma_{I I}=0.5 \mathrm{~J} / \mathrm{m}^{2}$. The effect of mode mix is neglected here by setting the shear strength and toughness identical to those for the opening mode.

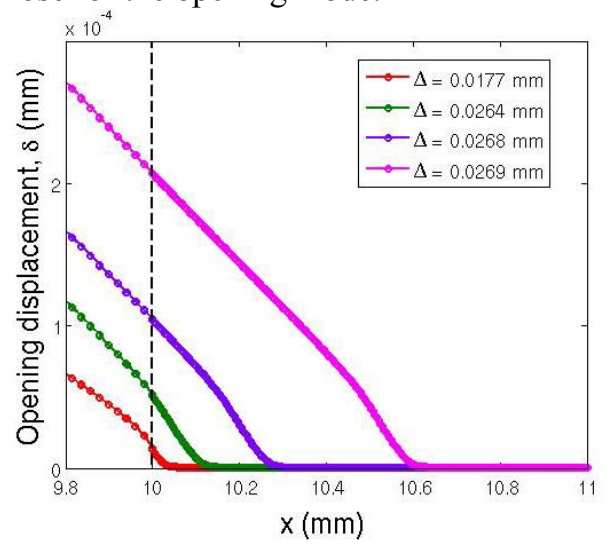

Fig. 10: Evolution of the opening displacement along the Si-Si interface for a simulated 4-point carrier bend test. The initial crack tip is at $x=10 \mathrm{~mm}$ (vertical dashed line), and the horizontal dashed line indicates the critical opening displacement $\left(\delta_{c}=0.5 \times 10^{-4} \mathrm{~mm}\right)$.

Figure 10 plots the opening displacements of the interface near the initial crack tip $\left(x=a_{0}=10 \mathrm{~mm}\right.$, measured from the left end of the sandwich specimen in Fig. 9) as the loading displacement $\Delta$ increases. Upon loading, the crack opens up along with the interface elements ahead of the crack tip $(x>$ $10 \mathrm{~mm}$ ). Following the bilinear traction-separation law, the interface opens elastically until it reaches the critical displacement, $\delta_{0}=\sigma_{0} / K_{0}=0.02 \times 10^{-4} \mathrm{~mm}$. Beyond $\delta_{0}$, the 
damage parameter $(D)$ of the interface element evolves from 0 to 1 , until it is fully damaged $(D=1)$. The critical opening displacement for a fully damaged interface element is, $\delta_{c}=2 \Gamma_{I} / \sigma_{0}=0.5 \times 10^{-4} \mathrm{~mm}$. As shown in Fig. 10, the opening displacement of the first interface element at $x=10$ mm reaches $\delta_{c}$ at $\Delta=0.0264 \mathrm{~mm}$, at which point the initial crack tip advances by one interface element. Ahead of the crack tip is a cohesive zone with partially damaged interface elements. Subsequently, as $\Delta$ increases, the crack grows with a steady-state cohesive zone. At each loading displacement, the crack grows stably and arrests at a crack length depending on both the toughness and strength of the interface. An infrared crack-opening interferometry (IR-COI) has been developed to measure the crack opening displacements near the crack tip, from which the traction-separation law of the interface can be deduced.

\section{SUMMARY}

This paper presents a nonlinear cohesive interface model to simulate initiation and propagation of interfacial delamination, which is applicable for the integrated thin-film structures in the chip-package systems. The method is demonstrated by two examples. The results show that in general both the strength and toughness play important roles in crack initiation and growth along an interface. Therefore, it is essential to develop experimental methods to characterize the detailed tractionseparation laws of specific interfaces.

\section{Acknowledgments}

The authors are grateful for the financial support by Semiconductor Research Corporation.

\section{References}

[1] P.S. Ho, G. Wang, M. Ding, J.H. Zhao, X. Dai, Microelectronics Reliability 44, 719-737 (2004).

[2] X.H. Liu, M.W. Lane, T.M. Shaw, E. Simonyi, Int. J. Solids and structures 44, 1706-1718 (2007).

[3] I. Mohammed and K.M. Liechti, J. Mechanics and Physics of Solids 48, 735-764 (2000).

[4] A.V. Mello, K.M. Liechti, J. Applied Mechanics 73, 860-870 (2006).

[5] Y. Zhu, K.M. Liechti, K. Ravi-Chandar, Int. J. Solids and Structures 46, 31-51 (2009).

[6] D.S. Dugdale, J. Appl. Mech. 8, 100-104 (1960).

[7] G.I. Barenblatt, Adv. Appl. Mech. 7, 55-129 (1962).

[8] A.H. Cottrell, Tewksbury Symposium on Fracture, 1-27 (1963).

[9] G. Bao and Z. Suo, Appl. Mech. Rev. 45, 355-366 (1992).

[10] J.W. Hutchinson and A.G. Evans, Acta Mater. 48, 125135 (2000).

[11] B.N. Cox and D.B. Marshall, Int. J. Fracture 49, 159-176 (1991).

[12] B.F. Sorensen, T.K. Jacobsen, Eng. Fracture Mech. 70, 1841-1858 (2003).

[13] J.P. Parmigiani and M.D. Thouless, Eng. Fracture Mech. 74, 2675-2699 (2007).
[14] C.G. Davila, P.P. Camanho, M.F. de Moura, AIAA-011486 (2001)

[15] M. Lane, R.H. Dauskardt, A. Vaichtein. H. Gao, J. Mater. Res. 15, 2758-2769 (2000).

[16] ABAQUS (version 6.8), Providence, RI.

[17] J.R. Rice, J. Appl. Mech. 55, 98-103 (1988).

[18] J.W. Hutchinson, Z. Suo, Advances in Applied Mechanics 29, 63-191 (1992).

[19] T. Ye, Z. Suo, A.G. Evans, J. Solids Struct., 29, 26392648 (1992).

[20] H. Mei, Y. Pang, R. Huang, Int. J. Fracture, 148, 331342 (2007). 